\title{
The histologic risk model is a useful and inexpensive tool to assess risk of recurrence and death in stage I or II squamous cell carcinoma of tongue and floor of mouth
}

\author{
Namita Sinha ${ }^{1}$, Matthew H Rigby ${ }^{2}$, Michael L McNeil ${ }^{2}$, S Mark Taylor ${ }^{2}$, Jonathan RB Trites ${ }^{2}$, \\ Robert D Hart ${ }^{2}$ and Martin J Bullock ${ }^{1}$ \\ ${ }^{1}$ Department of Pathology, Queen Elizabeth II Health Sciences Centre, Dalhousie University, Halifax, Nova \\ Scotia, Canada and ${ }^{2}$ Division of Otolaryngology-Head and Neck Surgery, Department of Surgery, Queen \\ Elizabeth II Health Sciences Centre, Dalhousie University, Halifax, Nova Scotia, Canada
}

\begin{abstract}
Surgery is the mainstay of treatment for low-stage (stage $1 / \mathrm{Il}$, ie, T1N0/T2N0) squamous cell carcinoma of oral cavity. However, a significant percentage of low-stage squamous cell carcinoma of oral cavity will develop local recurrence and disease-related mortality. In this study, we stratified 64 patients with low-stage of oral tongue and floor of mouth patients into high-, intermediate- and low-risk categories based on existing histologic risk model. The classification of these risk categories was based on presence or absence of perineural invasion and evaluation of tumor-host junction for worst pattern of invasion and lymphocytic host response. We correlated risk category and other variables with recurrence and death. In a univariate model, high-risk category tumors had a significantly higher rate of recurrence and death due to recurrence compared with low/intermediate-risk categories $(P=0.000$ and $P=0.047$, respectively). Controlling for margin status and T-stage, high-risk category had a 12.4 odds ratio of later recurrence when compared with low/intermediate-risk categories, with a $P$-value of 0.001. In conclusion, we found low-stage oral cavity squamous cell carcinoma patients with high-risk category have a significantly higher risk for recurrence when compared with patients in the low- or intermediate-risk category, even when controlling for margin status and T-stage. These patients may be suitable candidates for adjuvant treatment to decrease morbidity and mortality associated with a recurrence. Our results indicate that the histologic risk model is a useful and simple tool to assess risk of recurrence in stage I or II squamous cell carcinoma of oral cavity.
\end{abstract}

Modern Pathology (2018) 31, 772-779; doi:10.1038/modpathol.2017.183; published online 2 February 2018

Squamous cell carcinoma is the most common head and neck carcinoma, comprising $>90 \%$ of cancers of the head and neck region. ${ }^{1}$ Of all head and neck squamous cell carcinoma, squamous cell carcinoma of the oral cavity is the most common and is the sixth most common cancer worldwide. ${ }^{2}$ With the advancement in treatment modalities, survival has improved in locally advanced carcinoma of the tongue and oral cavity, with an increase in 5-year relative survival from $54 \%$ for the period $1982-1986$ to $62 \%$ for the

Correspondence: Dr N Sinha, MD, Department of Pathology and Immunology, Washington University, Washington University School of Medicine, 425 S. Euclid Avenue, Box 8118, St Louis, MO 63110, USA.

E-mail: drnamitasinha1@gmail.com

Received 4 July 2017; revised 8 November 2017; accepted 9 November 2017; published online 2 February 2018 period 2002-2006. ${ }^{3}$ However, despite increased detection of early-stage oral cavity squamous cell carcinoma, disease-specific mortality rates for stages I and II oral cavity squamous cell carcinoma remain at $25 \%$ and $37 \%$, respectively. ${ }^{4-6}$ Local control is a critical determinant of survival in early-stage oral cavity squamous cell carcinoma. ${ }^{6,7}$

Early-stage oral cavity squamous cell carcinoma are stage I/II (AJCC 7th edition categories, T1No/ T2N0), implying tumor size of $<4 \mathrm{~cm}$, with no lymph node or distant metastasis. Surgery continues to be the mainstay of treatment for early-stage oral cavity squamous cell carcinoma and postoperative adjuvant therapy is needed in patients with latestage oral cavity squamous cell carcinoma (stage III/ IV) or with adverse prognostic factors, such as poor differentiation, positive margins or advanced neck disease. ${ }^{8}$ About $30 \%$ of early-stage oral cavity 
squamous cell carcinoma patients do develop treatment failure and locoregional recurrence, indicating that more aggressive protocols are warranted for a subset of these patients. ${ }^{9}$ Positive surgical margins are known to govern local control rates. ${ }^{5}$ However, previous studies have shown that a significant percentage of margin-negative oral cavity squamous cell carcinoma do show treatment failure. Owing to easy access and visualization of the oral cavity structures, it is quite feasible to obtain clear margins in cancers of tongue and floor of mouth. Regardless of a clear margin, the treatment failure rate for earlystage oral cavity squamous cell carcinoma in our institution is $26 \%$, which is within the reported range of $10-40 \% .^{9}$

In the past, to predict the biological behavior of oral cavity squamous cell carcinoma, many workers

Table 1 Demographics and clinicopathologic characteristics of all patients with early-stage OSCC

Total number of patients

Site

\begin{tabular}{|c|c|}
\hline \multirow{2}{*}{\multicolumn{2}{|c|}{ Sex and age }} \\
\hline & \\
\hline Male & $\begin{array}{c}37(57 \%) \text {; age range of } 27-81 \\
\text { years }\end{array}$ \\
\hline Female & $28(43 \%) ;$ age range of $40-95$ \\
\hline \multicolumn{2}{|l|}{ Resection margin status } \\
\hline $\begin{array}{l}\text { Negative }(\geq 5 \mathrm{~mm}) \text {, close } \\
(<5 \mathrm{~mm})\end{array}$ & $57(88 \%)$ \\
\hline Positive & $8(12 \%)$ \\
\hline \multicolumn{2}{|l|}{ Tumor thickness } \\
\hline$\leq 4 \mathrm{~mm}$ & $13(20 \%)$ \\
\hline$>4 \mathrm{~mm}$ & $52(80 \%)$ \\
\hline \multicolumn{2}{|l|}{ Stage } \\
\hline Stage I (T1No) & $27(42 \%)$ \\
\hline Stage II (T2No) & $38(59 \%)$ \\
\hline Follow-up duration in months & $\begin{array}{l}24 \text { to } 168 \text { (mean }= \\
66 \text { months) }\end{array}$ \\
\hline Locoregional recurrence & $20(31 \%)$ \\
\hline Number of patients who died & $19(29 \%)$ \\
\hline Death related to disease & $11(17 \%)$ \\
\hline \multicolumn{2}{|l|}{ Worst pattern of invasion (WPOI) } \\
\hline $\begin{array}{l}\text { Nonaggressive, WPOI 1-3 } \\
\text { (score 0) }\end{array}$ & $26(40 \%)$ \\
\hline WPOI4 (score 1) & $24(37 \%)$ \\
\hline WPOI5 (score 3) & $15(23 \%)$ \\
\hline \multicolumn{2}{|l|}{ Lymphocytic host response (LHR) } \\
\hline Strong (score 0) & $7(11 \%)$ \\
\hline Intermediate (score 1) & $39(60 \%)$ \\
\hline Limited (score 3) & $19(29 \%)$ \\
\hline \multicolumn{2}{|l|}{ Perineural invasion (PNI) } \\
\hline None (score 0) & $27(42 \%)$ \\
\hline Small nerves (score 1) & $30(46 \%)$ \\
\hline Large nerves (score 3) & $8(12 \%)$ \\
\hline \multicolumn{2}{|l|}{ Risk category } \\
\hline Low & $5(8 \%)$ \\
\hline Intermediate & $26(40 \%)$ \\
\hline High & $34(52 \%)$ \\
\hline
\end{tabular}

have devised histological grading systems based on numerous features, including nuclear pleomorphism, mitotic index, lymphocytic response, tumor growth pattern, tumor thickness, histologic grade, depth of invasion, pattern of invasion, lymphovascular invasion and perineural invasion. ${ }^{10}$ Among these histological grading systems, BrandweinGensler's histologic risk model is the most popular. ${ }^{10,11}$ The histologic risk model was recently validated in a new patient cohort in 2010 to predict the risk for locoregional recurrence. ${ }^{11,12}$

In order to address the significant treatment failure rate in early-stage oral cavity squamous cell carcinoma in our institution, we applied the histologic risk model to a series of early-stage oral cavity squamous cell carcinoma to test if this model could predict which patients are at high risk for treatment failure.

\section{Materials and methods}

For the validation of the histologic risk model in our institution, with the approval of institutional ethics board, we retrospectively reviewed early-stage oral cavity squamous cell carcinoma (squamous cell carcinoma of the oral tongue and floor of mouth) with surgical treatment at our institute between January 2001 and December 2013.

The inclusion criteria for this study were: (1) primary surgeries with curative intent of histologically proven, clinically and pathologically earlystage squamous cell carcinoma of the oral tongue and floor of mouth; (2) generously sampled tumor-host interface (submitted either entirely or nearly entirely); and (3) minimum follow-up of 24 months, or to recurrence or death. The exclusion criteria were (1) node positive or late-stage squamous cell carcinoma (stage III/IV); (2) in-situ squamous cell carcinoma and microinvasive squamous cell carcinoma, with invasion of $2 \mathrm{~mm}$ or less in depth; and (3) spindle cell variant of squamous cell carcinoma and verrucous carcinoma. Although verrucous carcinoma was included in Brandwein-Gensler's 2013 risk model study, it was excluded from our study because of its distinct behavior (no risk for lymph node or distant metastasis) and rarity.

After review of the entire case of early-stage oral cavity squamous cell carcinoma that met our study criteria, we re-studied all the hematoxylin and eosin (H\&E)-stained pathology slides with sections containing tumor-host interface and/or perineural invasion. All cases were scored by a head and neck pathologist (MJB) and a senior pathology resident (NS) based on the published guidelines of the interpretation and application of the histologic risk model. ${ }^{9,11,12}$

Data collection included clinicopathologic and demographic information including age, gender, tumor site, $\mathrm{T}$ and $\mathrm{N}$ categories, pathology-related variables (tumor thickness, size, margin status, 
perineural invasion and histologic features at the tumor-host interface), and follow-up status, including tumor recurrence and death related to disease (Table 1). All information was updated and verified through medical records in December 2015 to include date of recurrence and death. Patients who were lost to follow-up were censored at the date last known to be alive.

Tumor thicknesses (in $\mathrm{mm}$ ) were recorded on all carcinomas. Resection margins were histologically assessed as follows: margins $\geq 5 \mathrm{~mm}$ from carcinoma were deemed negative; margins $<5 \mathrm{~mm}$ were deemed close and positive margins represented tumor at the inked resection margin.

The histologic risk model was applied as per instructions provided in the seminal publications and with advice from the author (MJB personal communication). ${ }^{12}$ The tumor was categorized according to risk level based on scoring ( 0,1 or 3$)$ of three variables, which included the worst pattern of tumor invasion, lymphocytic host response at the tumor-host interface and perineural invasion. ${ }^{9}$

Five patterns of invasion have been defined to denote the manner in which cancer infiltrates tissues at the tumor-host interface (Table 2,Figures 1a and b) and the highest score for the worst pattern of invasion was recorded; scores were 0 for worst pattern of invasion types 1-3, 1 for worst pattern of invasion type 4 and 3 for worst pattern of invasion type 5 . Worst pattern of invasion type 5 is also referred to as multifocal pattern as it contains dispersed tumor satellites, with at least $1 \mathrm{~mm}$ of intervening normal tissue between at least two tumor masses (Figure 1a). Three patterns of lymphocytic host response at the tumor-host interface and perineural invasion are defined in Table 2. Nerves were measured in cross-section only. The sum of the assigned scores for all three variables were used to compute the risk category as low risk for score 0 , intermediate risk for scores 1-2 and high risk for scores 3-9. The low- and intermediate-risk categories were combined for comparative analysis with the high-risk category group.

Of note, the pathologists did not receive any special training in histologic risk model interpretation or application. However, histologic risk model was applied by strictly following guidelines from Brandwein-Gensler's previous published papers. ${ }^{9,11-12}$ The pathologists were also blinded to the patient's outcomes.

The primary endpoints for this study were locoregional recurrence, distant metastasis and disease-related death. Locoregional recurrence includes local recurrence (defined as biopsy proven tumor at the original primary location) and/or regional lymph node metastasis. Disease-related death was defined as death from oral cancer or the direct effects of its treatment.

Statistical analysis was performed using SPSS 22 software (IBM Corp, Armonk, NY, USA). Analyses were applied for risk category, the worst pattern of invasion score, perineural invasion, lymphocytic host response, multifocality, tumor thickness, tumor stage and margin status. Univariate association between these factors and outcomes were tested using a two-tailed Fisher's exact test. In addition, multivariate analysis was performed using a logistic regression model to assess the association of recurrence with T-stage (1 vs 2), margin status and risk category (high vs low/intermediate). Statistical significance was claimed at $\leq 0.05$.

\section{Results}

A total of 65 patients with oral cavity squamous cell carcinoma fulfilled our inclusion criteria and were assigned risk categories according to histologic risk model. Table 1 summarizes the demographic and clinicopathologic features of the patients. The patient population comprised 37 males and 28 females with ages ranging from 27 to 81 years for men and 40 to 95

Table 2 Brandwein-Gensler's risk scoring system ${ }^{9,10}$

\begin{tabular}{|c|c|c|}
\hline Variable & Definition & Point assignment \\
\hline \multicolumn{2}{|c|}{ Worst pattern of Invasion (WPOI) } & WPOI score \\
\hline Type 1 & Pushing border (Figure 1b) & 0 \\
\hline Type 2 & Finger-like growth & 0 \\
\hline Type 3 & Large separate islands, $>15$ cells per island & 0 \\
\hline Type 4 & Small tumor islands, 15 cells or fewer, per island & +1 \\
\hline Type 5 & Tumor satellites, $=/>1 \mathrm{~mm}$ from main tumor or next closest satellite (Figure 1a) & +3 \\
\hline \multicolumn{2}{|c|}{ Lymphocytic host response (LHR) } & LHR score \\
\hline Type 1 & Dense complete host response rimming tumor (Figure 1c) & 0 \\
\hline Type 2 & Lymphoid nodules in some but not all $4 \times$ fields & +1 \\
\hline Type 3 & Little or no host response (Figure $1 \mathrm{~b}$ ) & +3 \\
\hline \multicolumn{2}{|c|}{ Perineural invasion (PNI) } & PNI score \\
\hline None & None & 0 \\
\hline Small nerve & Tumor wrapping around nerves, $<1 \mathrm{~mm}$ diameter & +1 \\
\hline Large nerve & Tumor wrapping around nerves, $=/>1 \mathrm{~mm}$ diameter (Figure $1 \mathrm{~d}$ ) & +3 \\
\hline
\end{tabular}



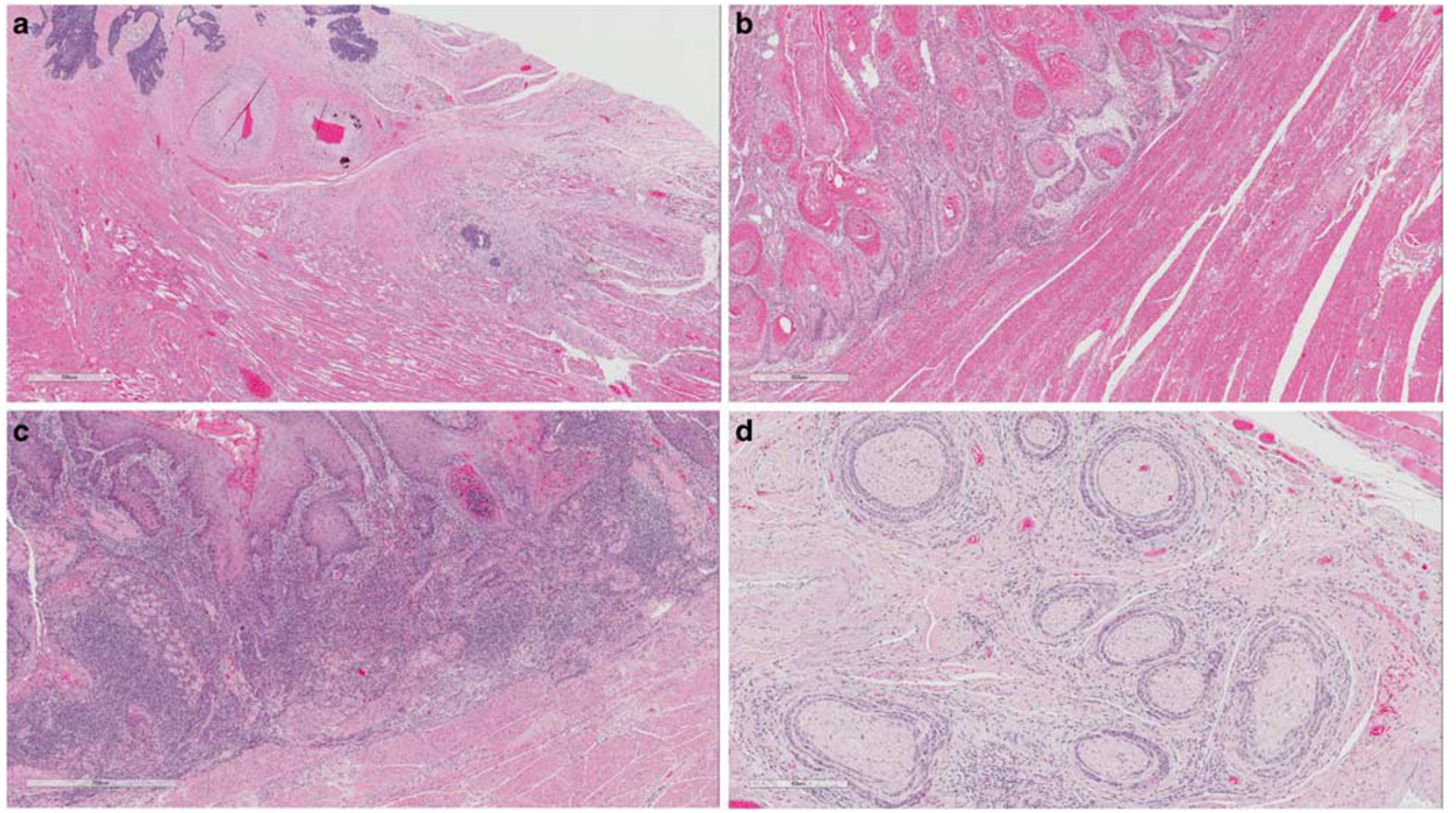

Figure 1 Representative low to medium power views of H\&E-stained sections of oral squamous cell carcinoma showing: tumor satellite at $1 \mathrm{~mm}$ or greater from main tumor with intervening normal tissue (worst pattern of invasion type 5) (a); tumor invading in a broad pushing manner (worst pattern of invasion type 1), with no significant lymphocytic host response (lymphocytic host response type 3) (b); continuous dense rim of lymphoid tissue at the tumor-host interface (lymphocytic host response type 1) (c); and perineural invasion of a large nerve (d).

years for women. All patients were immunocompetent, with the exception of one patient who had Fanconi anemia and one who was HIV-positive and stable on antiretroviral drugs for many years. There were $27(42 \%)$ patients in stage I (pT1No) and 38 $(59 \%)$ patients in stage II (pT2No). None of the patients received neoadjuvant chemotherapy and/or radiation therapy. Only two patients received adjuvant radiotherapy, one for positive margins and one for a close margin of $<1 \mathrm{~mm}$.

Mean follow-up for all survivors was 66 months, with a range of $24-168$ months. In all, $46(71 \%)$ patients were alive, of whom $37(57 \%)$ were disease free and $9(14 \%)$ were alive with disease. A total of 19 patients (29\%) died; 11 (18\%) from oral cavity squamous cell carcinoma-related causes (diseaserelated death) and 8 patients (12\%) from unrelated causes. No patient developed distant metastasis as a first disease progression event.

Of the 65 evaluable patients for histologic risk model, $5(8 \%)$ were classified as low-risk category (score 0), $26(40 \%)$ as intermediate-risk category (scores 1-2) and 34 (52\%) as high-risk category (scores 3-9). The HIV-positive patient and Fanconi anemia patient both had high-risk category tumors. The number of high-risk category tumors was significantly higher among the stage II tumors, (66\%) compared with stage I tumors $(33 \%)(P=0.013)$, which was primarily because of higher lymphocytic host response
$(P=0.011)$ and perineural invasion $(P=0.02)$ categories (Table 3). However, there were no significant difference in the incidence of locoregional recurrence $(P=0.278)$ or disease-related death $(P=0.751)$ between patients with stage I and II tumors.

Univariate analysis demonstrated that locoregional recurrence was significantly associated with higher worst pattern of invasion score (score $3 \mathrm{vs}$ $0 / 1)(P=0.032)$, presence of perineural invasion $(P=0.028)$ and high-risk category $(P=0.000)$, but not with lymphocytic host response score $(P=0.423)$ or multifocality/worst pattern of invasion type 5 $(P=0.524)$. Disease-related death was significantly associated with high-risk category $(P=0.047)$, but not with worst pattern of invasion type 4 and $5(P=0.177)$, presence of perineural invasion $(P=0.105)$, lymphocytic host response $(P=1.000)$ or multifocality $(P=0.261)$ (Tables 4,5,6,7,8).

Margins were recorded as negative or close in 57 patients $(88 \%)$ and positive in 8 patients $(12 \%)$. Local recurrence was observed in 20 of 65 patients (31\%), of which three occurred in patients with positive margins. Disease-related death was observed in 11 of 65 patients (17\%); of these deaths, two occurred in patients with positive margins. However, there was no significant difference in locoregional recurrence and disease-related death in patients with positive margins vs negative margins (locoregional recurrence-38\% (3/8) vs $30 \%$ (17/57), $P=0.693$; 
Table 3 Tumor stage: relationship to recurrence and death

\begin{tabular}{|c|c|c|c|c|c|c|c|}
\hline \multirow[t]{2}{*}{ TNM stage } & \multicolumn{2}{|c|}{ Risk category } & \multicolumn{2}{|c|}{ Recurrence } & \multicolumn{2}{|c|}{ Death due to disease } & \multirow[t]{2}{*}{$\begin{array}{c}\text { No. of patients }(\mathrm{n}=65 \text {, } \\
100 \%)\end{array}$} \\
\hline & $\begin{array}{c}\text { Low/ } \\
\text { intermediate } \\
(\mathrm{n}=31,48 \%)\end{array}$ & $\begin{array}{c}\operatorname{High}(\mathrm{n}=34 \\
52 \%)\end{array}$ & $\begin{array}{c}\text { No. }(\mathrm{n}=45 \text {, } \\
69 \%)\end{array}$ & $\begin{array}{c}\text { Yes }(\mathrm{n}=20 \\
31 \%)\end{array}$ & $\begin{array}{c}\text { No. }(\mathrm{n}=54 \\
83 \%)\end{array}$ & $\begin{array}{c}\text { Yes }(\mathrm{n}=11 \\
17 \%)\end{array}$ & \\
\hline I (pT1N0) & $18(67 \%)$ & $9(33 \%)$ & $21(78 \%)$ & $6(22 \%)$ & $23(85 \%)$ & $4(15 \%)$ & $27(88 \%)$ \\
\hline \multirow{2}{*}{ II (pT2No) } & $13(34 \%)$ & $25(66 \%)$ & $24(63 \%)$ & $14(37 \%)$ & $31(82 \%)$ & $7(18 \%)$ & $38(13 \%)$ \\
\hline & \multicolumn{2}{|c|}{$P$-value $=0.013$} & \multicolumn{2}{|c|}{$P$-value $=0.278$} & \multicolumn{2}{|c|}{$P$-value $=0.751$} & \\
\hline
\end{tabular}

Table 4 Worst pattern of Invasion (WPOI): relationship to recurrence and death

\begin{tabular}{|c|c|c|c|c|c|}
\hline \multirow[t]{2}{*}{ WPOI score } & \multicolumn{2}{|c|}{ Recurrence } & \multicolumn{2}{|c|}{ Death due to disease } & \multirow[t]{2}{*}{ No. of patients $(\mathrm{n}=65,100 \%$, } \\
\hline & $N o(\mathrm{n}=45,69 \%)$ & Yes $(\mathrm{n}=20,31 \%)$ & No $(\mathrm{n}=54,83 \%)$ & Yes $(\mathrm{n}=11,17 \%)$ & \\
\hline 0 & $22(85 \%)$ & $4(15 \%)$ & $24(92 \%)$ & $2(7 \%)$ & $26(40 \%)$ \\
\hline \multirow[t]{2}{*}{$1 / 3$} & $23(59 \%)$ & $16(41 \%)$ & $30(77 \%)$ & $9(23 \%)$ & $39(60 \%)$ \\
\hline & \multicolumn{2}{|c|}{$P$-value $=0.032$} & \multicolumn{2}{|c|}{$P$-value $=0.177$} & \\
\hline
\end{tabular}

Table 5 LHR: relationship to recurrence and death

\begin{tabular}{|c|c|c|c|c|c|}
\hline \multirow[t]{2}{*}{ LHR score } & \multicolumn{2}{|c|}{ Recurrence } & \multicolumn{2}{|c|}{ Death due to disease } & \multirow[t]{2}{*}{ No. of patients $(\mathrm{n}=65,100 \%)$} \\
\hline & No $(\mathrm{n}=45,69 \%)$ & Yes $(\mathrm{n}=20,31 \%)$ & $N o(\mathrm{n}=54,83 \%)$ & Yes $(\mathrm{n}=11,17 \%)$ & \\
\hline 0 & $6(86 \%)$ & $1(14 \%)$ & $6(86 \%)$ & $1(14 \%)$ & $7(11 \%)$ \\
\hline \multirow[t]{2}{*}{$1 / 3$} & $39(67 \%)$ & $19(33 \%)$ & $48(83 \%)$ & $10(17 \%)$ & $58(89 \%)$ \\
\hline & \multicolumn{2}{|c|}{$P$-value $=0.423$} & \multicolumn{2}{|c|}{$P$-value $=1.000$} & \\
\hline
\end{tabular}

Abbreviation: LHR, lymphocytic host response.

Table 6 Presence of PNI: relationship to recurrence and death

\begin{tabular}{|c|c|c|c|c|c|}
\hline \multirow[t]{2}{*}{ PNI score } & \multicolumn{2}{|c|}{ Recurrence } & \multicolumn{2}{|c|}{ Death due to disease } & \multirow[t]{2}{*}{ No. of patients $(\mathrm{n}=65,100 \%)$} \\
\hline & $N o(\mathrm{n}=45,69 \%)$ & Yes $(\mathrm{n}=20,31 \%)$ & $N o(\mathrm{n}=54,83 \%)$ & Yes $(\mathrm{n}=11,17 \%)$ & \\
\hline 0 & $23(85 \%)$ & $4(15 \%)$ & $25(93 \%)$ & $2(7 \%)$ & $27(42 \%)$ \\
\hline \multirow[t]{2}{*}{$1 / 3$} & $22(58 \%)$ & $16(42 \%)$ & $29(76 \%)$ & $9(24 \%)$ & $38(56 \%)$ \\
\hline & \multicolumn{2}{|c|}{$P$-value $=0.028$} & \multicolumn{2}{|c|}{$P$-value $=0.105$} & \\
\hline
\end{tabular}

Abbreviation: PNI, perineural invasion.

Table 7 Multifocality: relationship to recurrence and death

\begin{tabular}{|c|c|c|c|c|c|}
\hline \multirow[t]{2}{*}{ Multifocality } & \multicolumn{2}{|c|}{ Recurrence } & \multicolumn{2}{|c|}{ Death due to disease } & \multirow[t]{2}{*}{ No. of patients $(\mathrm{n}=65,100 \%)$} \\
\hline & $N o(\mathrm{n}=45,69 \%)$ & Yes $(\mathrm{n}=20,31 \%)$ & $N o(\mathrm{n}=54,83 \%)$ & Yes $(\mathrm{n}=11,17 \%)$ & \\
\hline No & $36(72 \%)$ & $14(28 \%)$ & $43(86 \%)$ & $7(14 \%)$ & $50(48 \%)$ \\
\hline \multirow[t]{2}{*}{ Yes } & $9(60 \%)$ & $6(40 \%)$ & $11(73 \%)$ & $4(27 \%)$ & $15(52 \%)$ \\
\hline & \multicolumn{2}{|c|}{$P$-value $=0.524$} & \multicolumn{2}{|c|}{$P$-value $=0.261$} & \\
\hline
\end{tabular}


Table 8 Risk category: relationship to recurrence and death

\begin{tabular}{|c|c|c|c|c|c|}
\hline \multirow[t]{2}{*}{ Risk category } & \multicolumn{2}{|c|}{ Recurrence } & \multicolumn{2}{|c|}{ Death due to disease } & \multirow[t]{2}{*}{ No. of patients $(\mathrm{n}=65,100 \%)$} \\
\hline & $N o(\mathrm{n}=45,69 \%)$ & Yes $(\mathrm{n}=20,31 \%)$ & No $(\mathrm{n}=54,83 \%)$ & Yes $(\mathrm{n}=11,17 \%)$ & \\
\hline Low/intermediate & $28(90 \%)$ & $3(10 \%)$ & $29(94 \%)$ & $2(7 \%)$ & $31(47 \%)$ \\
\hline High & $17(50 \%)$ & $17(50 \%)$ & $25(74 \%)$ & $9(27 \%)$ & $34(52 \%)$ \\
\hline & \multicolumn{2}{|c|}{$P$-value $=0.000$} & \multicolumn{2}{|c|}{$P$-value $=0.047$} & \\
\hline
\end{tabular}

Table 9 Margin status: relationship to recurrence and death

\begin{tabular}{|c|c|c|c|c|c|}
\hline \multirow[t]{2}{*}{ Margin status } & \multicolumn{2}{|c|}{ Recurrence } & \multicolumn{2}{|c|}{ Death due to disease } & \multirow[t]{2}{*}{ No. of patients $(\mathrm{n}=65,100 \%)$} \\
\hline & No $(\mathrm{n}=45,69 \%)$ & Yes $(\mathrm{n}=20,31 \%)$ & No $(\mathrm{n}=54,83 \%)$ & Yes $(\mathrm{n}=11,17 \%)$ & \\
\hline Negative & $40(70 \%)$ & $17(30 \%)$ & $48(84 \%)$ & $9(16 \%)$ & $57(88 \%)$ \\
\hline \multirow[t]{2}{*}{ Positive } & $5(63 \%)$ & $3(38 \%)$ & $6(75 \%)$ & $2(25 \%)$ & $8(13 \%)$ \\
\hline & \multicolumn{2}{|c|}{$P$-value $=0.693$} & \multicolumn{2}{|c|}{$P$-value $=0.614$} & \\
\hline
\end{tabular}

Table 10 Logistic regression analyzing odds ratio for recurrence in high risk category compared to low/intermediate risk category while controlling for T-stage, margin status and multifocality

\begin{tabular}{lrccc}
\hline Variables & $\beta$ & s.e. & P-value & OR \\
\hline $\begin{array}{l}\text { Risk category-high (vs low/ } \\
\text { intermediate) }\end{array}$ & 2.521 & 0.783 & 0.001 & 12.444 \\
T2 (vs T1) & & & & \\
Positive margin & 0.183 & 0.670 & 0.784 & 1.201 \\
Multifocal & -0.190 & 0.876 & 0.829 & 0.827 \\
Constant & -0.704 & 0.734 & 0.337 & 0.494 \\
& -2.491 & 1.148 & 0.030 & 0.083 \\
\hline
\end{tabular}

Table 11 Logistic regression analyzing odds ratio for diseaserelated death in high risk category compared to low/intermediate risk category while controlling for T-stage, margin status and multifocality

\begin{tabular}{lrccc}
\hline Variables & $\beta$ & s.e. & P-value & OR \\
\hline $\begin{array}{l}\text { Risk category_high (vs low/ } \\
\text { intermediate) }\end{array}$ & 1.713 & 0.929 & 0.065 & 5.546 \\
T2 (vs T1) & -0.262 & 0.754 & 0.728 & 0.769 \\
Positive margin & 0.061 & 0.972 & 0.950 & 1.063 \\
Multifocal & 0.034 & 0.824 & 0.967 & 1.035 \\
Constant & -2.311 & 1.264 & 0.067 & 0.099 \\
\hline
\end{tabular}

disease-related death-25\% (2/8) vs $16 \%$ (9/57), $P=0.614$ ) (Table 9).

The multivariate analysis demonstrated that in node-negative $\mathrm{T} 1$ and $\mathrm{T} 2$ oral cavity squamous cell carcinoma, controlling for T-stage and margin status, the odds ratio of recurrence for the high-risk category was 12.4 when compared with low- or intermediaterisk categories. This is statistically significant at a level of 0.001 (Table 10). However, the odds ratio of 5.55 for the association with risk of death due to recurrence for high-risk category compared with low- or intermediate-risk categories did not reach statistical significance $(P=0.065)$ (Table 11$)$.

\section{Discussion}

In 2007, the 5-year disease-specific survival rate for patients with oral tongue cancer in the SEER registry was $60 \%$, reflecting only a marginal improvement in the past 20 years. ${ }^{12}$ However, over the past decade, the 5-year survival rate has improved for advanced head and neck squamous cell carcinoma. ${ }^{3}$ A period analysis using a large SEER database for head and neck squamous cell carcinoma demonstrated that the greatest survival improvements were in locally advanced carcinoma of the tongue and oral cavity cancers. This was attributed to use of adjuvant chemo-radiation in these patients. ${ }^{3,13}$

Currently, treatment is usually intensified for advanced tumors, while margin-negative early-stage oral cavity squamous cell carcinoma continues to undergo surgical resection, often with no adjuvant therapy, regardless of inherent differences in tumor biology. ${ }^{5}$ Previous studies have shown that oral cavity squamous cell carcinoma has been associated with several molecular abnormalities; however, no specific marker has been shown to uniformly and reliably predict tumor behavior. ${ }^{14,15}$ The utility of molecular assays in the clinical settings is further limited by cost and time issues. ${ }^{14}$ On the other hand, a histopathology-based scoring system is easy to apply on H\&E-stained sections from an adequately sampled tumor-host interface. Based on the study of various histological parameters and assessment of tumor-host junction on routine H\&E stains, Brandwein-Gensler developed and validated a predictive model for patients with head and neck squamous 
cell carcinoma referred to as the risk model. ${ }^{9,11-12}$ Presently, this is the only validated predictor of treatment failure for patients with early-stage head and neck squamous cell carcinoma. ${ }^{12}$

With the application of histologic risk model to early-stage oral cavity squamous cell carcinoma in our institution, we stratified the risk categories further high-risk category vs low- or intermediaterisk categories. Our analysis demonstrated that highrisk category significantly correlated with high treatment failure rate (locoregional recurrence and disease-related death) in a univariate analysis. Locoregional recurrence and disease-related death occurred in $31 \%(n=20)$ and $17 \%(n=11)$ patients, respectively. The locoregional recurrence and disease-related death were significantly higher in high-risk category compared with low/intermediaterisk categories ( $P=0.000$ and 0.047 , respectively) (Table 8). Our findings support using the histologic risk model as a tool to predict tumor behavior in patients with early-stage oral cavity squamous cell carcinoma.

Brandwein-Gensler's histologic risk model is the first validated model that is significantly predictive for the important niche group of low-stage oral cavity squamous cell carcinoma patients. ${ }^{12}$ Based on multivariable analysis of 299 patients, the risk model was significantly predictive of locoregional recurrence and disease-specific survival. ${ }^{12}$ In Brandwein-Gensler's study, high-risk classification for a combination of features other than worst pattern of invasion type 5 had $32 \%$ probability of developing locoregional recurrence. Worst pattern of invasion alone was also significantly predictive for locoregional recurrence and disease-specific survival, with $42 \%$ probability of developing locoregional recurrence in worst pattern of invasion type $5 .^{12}$ In our study, both disease-related death and locoregional recurrence was significantly associated with high-risk category but not with multifocality/worst pattern of invasion type 5. We have no clear explanation for the discrepancy between our results and BrandweinGensler's. At the time of treatment, the worst pattern of invasion was unknown to the treating physicians as it had not been calculated, and so it should have had no effect on treatment (eg, width of margins resected or decisions to give adjuvant treatment). The rate of local recurrence among patients with worst pattern of invasion type 5 in our study was $40 \%$, which was similar to the 2013 BrandweinGensler study. However, although the combined percentage of worst pattern of invasion type 4 and 5 cases of the total was similar in our study and their study (60\% vs $67 \%$, respectively), they had a $16 \%$ rate of worst pattern of invasion type 5 and $51 \%$ rate of worst pattern of invasion type 4 vs our $23 \%$ and $37 \%$. It is possible that we applied the criteria for distinction between worst pattern of invasion type 4 and 5 differently. However, we speculate that patients with worst pattern of invasion type 4 are also at high risk for recurrence. In our study, there was a significant difference in the rate of recurrence between those with combined worst pattern of invasion type 4 and 5 verses those with worst pattern of invasion type $1-3(P=0.032$, see Table 5$)$, which supports that contention. Most patients with worst pattern of invasion type 4 would be in the high-risk category.

A similar study, in a tightly controlled cohort of 60 transoral laser microsurgery-treated margin-negative early-stage oral cavity squamous cell carcinoma, reported poorer local control and disease-specific survival in the histologic risk model determined high-risk group. ${ }^{16}$ In addition, these authors demonstrated that the presence of immune compromise was the strongest predictor for overall survival in patients with oral cavity squamous cell carcinoma. ${ }^{16}$ In our study, only 2 of 65 patients (3\%) were immunocompromised, both of whom had high-risk category tumors. However, because of the small numbers involved, we did not include this factor in our statistical calculations.

In univariate analysis, we also observed that the primary tumor thickness ( $>4 \mathrm{~mm}$ ), worst pattern of invasion score ( 1 or 3 vs 0 ) and presence of perineural invasion predicted a greater risk of neck recurrence, but not death due to disease. T-stage (T1 vs T2), margin status, multifocality and lymphocytic host response did not show a statistically significant association with increased risk of recurrence or death due to disease. Of note, the rate of high-risk category was significantly higher in stage II patients $(68 \%)$ compared with stage I patients $(33 \%)$ $(P=0.013)$. Further analysis revealed that the higher rate of high-risk category in stage II cases was attributed to the presence of perineural invasion and poor lymphocytic host response in these patients. These findings indicate that early-stage oral cavity squamous cell carcinoma, especially of size 2$4 \mathrm{~cm}$ (T2 tumors) should be very carefully evaluated for the high-risk features at the tumor-host interface and for perineural invasion.

In margin-negative oral cavity squamous cell carcinoma, the rates of local recurrence and disease-related death were $30 \%$ and $16 \%$, respectively. Our analyses did not demonstrate any negative impact of positive margins on local control or survival outcome (Table 3). In a retrospective study of surgically treated squamous cell carcinoma of the tongue and floor of the mouth by Weijers et $a l^{17}$, a comparison between local recurrences in patients with free surgical margins $(\geq 5 \mathrm{~mm})$ and patients with close/positive surgical margins $(<0.5 \mathrm{~mm})$ did not show a statistically significant difference. Using the same margin width $(<0.5 \mathrm{~mm})$ for squamous cell carcinoma of the floor of the mouth and the oral tongue, Zelefsky et $a l^{18}$ concluded that this prognostic factor did not have a significant impact on local control. On the other hand, Loree and Strong ${ }^{19}$ found that the local recurrence rate for the close/positive margin 
category $(<0.5 \mathrm{~mm}$ ) was significantly different from the negative margin rate. ${ }^{9}$

Our multivariate analysis indicates a strong association of local recurrence with high-risk category, irrespective of the margin status, indicating that more aggressive protocols may be warranted for this subset of early-stage oral cavity squamous cell carcinoma patients. Although the sample for this analysis was small, our data indicate that histologic risk model facilitates risk assessment in this group of patients, and would strongly support the routine use of the histologic risk model in reporting of earlystage oral cavity squamous cell carcinoma. This finding commensurate with other studies of the histologic risk model in this setting.

In conclusion, Brandwein-Gensler's histologic risk model is an easily applied, inexpensive and useful tool for risk assessment of node negative T1 and T2 oral cavity squamous cell carcinoma. In order to achieve this, early-stage oral cavity squamous cell carcinoma should be submitted completely at the tumor-host interface and be carefully evaluated for three histological factors (worst pattern of invasion, lymphocytic host response and perineural invasion) on routine histopathologic examination.

Early-stage oral cavity squamous cell carcinoma patients who are at high risk for disease progression based on the histologic risk model, may be considered for more aggressive protocols for the disease control.

\section{Disclosure/conflict of interest}

The authors declare no conflict of interest.

\section{References}

1 Parkin DM. Global cancer statistics in the year 2000. Lancet Oncol 2001;2:533-543.

2 Shah JP, Gil Z. Current concepts in management of oral cancer-surgery. Oral Oncol 2009;45:394-401.

3 Pulte D, Brenner H. Changes in survival in head and neck cancers in the late 20th and early 21st century: a period analysis. Oncologist 2010;15:994-1001.

4 Carvalho AL, Nishimoto IN, Califano JA, et al. Trends in incidence and prognosis for head and neck cancer in the United States: a site-specific analysis of the SEER database. Int J Cancer 2005;114:806-816.

5 Sutton DN, Brown JS, Rogers SN, et al. The prognostic implications of the surgical margin in oral squamous cell carcinoma. Int J Oral Maxillofac Surg 2003;32: 30-34.

6 Iseli TA, Lin MJ, Tsui A, et al. Are wider surgical margins needed for early oral tongue cancer? J Laryngol Otol 2012;126:289-294.

7 Rennemo E, Zatterstrom U, Boysen M. Outcome of local failures after oral cancer-recurrence vs. second primary. J Oral Pathol Med 2010;39:657-661.

8 Omura K. Current status of oral cancer treatment strategies: surgical treatments for oral squamous cell carcinoma. Int J Clin Oncol 2014;19:423-430.

9 Brandwein-Gensler M, Teixeira MS, Lewis CM, et al. Oral squamous cell carcinoma: histologic risk assessment, but not margin status, is strongly predictive of local disease-free and overall survival. Am J Surg Pathol 2005;29:167-178.

10 Bryne M, Koppang HS, Lilleng R, et al. New malignancy grading is a better prognostic indicator than Broders' grading in oral squamous cell carcinomas. J Oral Pathol Med 1989;18:432-437.

11 Brandwein-Gensler M, Smith RV, Wang B, et al. Validation of the histologic risk model in a new cohort of patients with head and neck squamous cell carcinoma. Am J Surg Pathol 2010;34:676-688.

12 Li Y, Bai S, Carroll W, et al. Validation of the risk model: high-risk classification and tumor pattern of invasion predict outcome for patients with low-stage oral cavity squamous cell carcinoma. Head and Neck Pathol 2013;7:211-223.

13 National Cancer InstituteSurveillance, Epidemiology, and End Results (SEER) web site http://seer.cancer.gov/ statistics. Accessed January 2011.

14 Braakhuis BJ, Bloemena E, Leemans CR, et al. Molecular analysis of surgical margins in head and neck cancer: more than a marginal issue. Oral Oncol 2010;46:485-491.

15 Curry JM, Sprandio J, Cognetti D, et al. Tumor microenvironment in head and neck squamous cell carcinoma. Semin Oncol 2014;41:217-234.

16 Sinha P, Mehrad M, Chernock RD, et al. Histologic and systemic prognosticators for local control and survival in margin-negative transoral laser microsurgery treated oral cavity squamous cell carcinoma. Head Neck 2015;37: 52-63.

17 Weijers M, Snow GB, Bezemer DP, et al. The status of the deep surgical margins in tongue and floor of mouth squamous cell carcinoma and risk of local recurrence; an analysis of 68 patients. Int J Oral Maxillofac Surg 2004;33:146-149.

18 Zelefsky MJ, Harrison LB, Fass DE, et al. Postoperative radiotherapy for oral cavity cancers: impact of anatomic subsite on treatment outcome. Head Neck 1990;12:470-475.

19 Loree TR, Strong EW. Significance of positive margins in oral cavity squamous carcinoma. Am J Surg 1990;160: $410-414$ 